\title{
Influence of decay in the target on the measurement of release times and release efficiency
}

\author{
G. Lhersonneau ${ }^{\mathrm{a}, *, 1}$, A.E. Barzakh ${ }^{\mathrm{b}}$, V. Rizzi ${ }^{\mathrm{a}}$, O. Alyakrinskiy ${ }^{\mathrm{a}}$, K.A. Mezilev ${ }^{\mathrm{b}}$, \\ F.V. Moroz ${ }^{\mathrm{b}}$, V.N. Panteleev ${ }^{\mathrm{b}}$, L.B. Tecchio ${ }^{\mathrm{a}}$ \\ "INFN, Laboratori Nazionali di Legnaro Viale dell'Università 2, 35020 Legnaro (PD). Italy. \\ betersburg Nuclear Physics Institute, Leningrad district, 18850 Gatchina, Russia
}

Available online 5 June 2008

\begin{abstract}
We present a framework for the experimental determination of release times and release efficiencies of ISOL-targets devoted to production of neutron-rich nuclei. Since a wide $(\mathrm{Z}, \mathrm{A})$ distribution of products is generated in fission, decay in the target of nuclei more exotic than the one investigated is an extra production channel. This feeding, not in phase with the accelerator beam, modifies the release curves. A model which explicitly takes into account the occurence of $\beta$ decay during diffusion in the grains or effusion between them has been designed. We also discuss the fact that in measurement of efficiency versus lifetime, an effective cross-section must be used which incorporates feedings by the mother nucleus and occasionally of isomers of the nucleus investigated. Release efficiency and release curves must be analysed in a consistent framework to obtain meaningful data.
\end{abstract}

(c) 2008 Elsevier B.V. All rights reserved.

PACS: $29.25 . \mathrm{Rm} ; 29.30 . \mathrm{Kv}$

Keywords: Release time from ISOL target: Decay in target

\section{Introduction}

On-line mass separation has been employed with impressive success for several decades for decay studies of artificially produced nuclei, see e.g. the review in [1]. For some time now, the isotope separation on-line (ISOL) method has also been considered as being the most efficient one for the first production stage of radioactive beam facilities (RIB) in operation or being planned. The tests carried out at the IRIS on-line mass separator at PNPI-Gatchina by the PLOG-collaboration (PNPI-Gatchina, LNLINFN-Legnaro, IPN-Orsay and GANIL) [2], aim at the future development of massive $\mathrm{UC}_{x}$ targets of circa $1 \mathrm{~kg}$ to be irradiated by neutrons of intermediate energy, with applications for the future radioactive-beam facilities

\footnotetext{
Corresponding author.

E-mail address: lhersonneau@ganil.fr (G. Lhersonneau).

${ }^{1}$ Now at GANIL, Caen, France.
}

SPES, SPIRAL-II and EURISOL [3-5]. Yet, a crucial problem is to ensure efficient release of the nuclei embedded in the target. Methods to measure release time distributions and formalism to extract parameters characterizing them have been developed over the years. However, to our knowledge, there does not exist a consistent treatment of decay in the target, and this issue is thus explored here. Part of this work, i.e. the distorsion of release curves, has been published [6] and shall be only outlined here, but release efficiency curves shall be discussed in some detail.

The release function $R(t)$ can be regarded as the response of the separator, i.e. as an ion current $i(t)$, to a short pulse of the accelerator at time $t=0$. In our experiments we have first to perform an irradiation of the target for a time long enough to reach a saturation of this current. Then, the accelerator beam is switched off and the ion current is observed as function of time. The resulting curve is the release curve. It is related to the release function by convolution with the in-target production rate. Decay in 
the target is irrelevant if the reactions to produce the nucleus of interest are such as $(p, x n)$ but it is especially important in our experiments. We indeed produce neutron-rich nuclei by fission induced by $1 \mathrm{GeV}$ protons. This creates a rather broad distribution of isobars.

The first part of the measurement, with beam on, corresponds to the conditions of operation of a RIB facility. The efficiency is derived from the current at the end of a long irradiation. It naturally supposes that the in-target production rate is known. Therefore, we must quantify how many of the produced more neutron-rich isobars actually decay in the target. As is well-known, variation of efficiency versus half-life of isotopes allows to extract release parameters, according to assumptions on the release mechanism.

The second part of the measurement, with accelerator beam off, yields the release curve. Here, too, decay in the target plays a role. It is a source of nuclei of interest, but with a creation rate not following the profile of the accelerator pulse. The shape of the release curve is therefore perturbed.

\section{The DBBM model}

\subsection{Description}

The 'Double-Black-Box' has been introduced in [6].

The model is shown schematically in Fig. 1. The vertical line on the left marks the time of creation by the reaction. The beam is assumed constant during irradiation for simplicity. Inside the grain of matter, at any time the atom can diffuse towards the surface (horizontal line) or change its nature through $\beta$ decay of its nucleus (vertical line). When diffusion is completed, i.e. the middle vertical line in Fig. 1 is reached, the calculation of effusion proceeds in the same way, except that the input rate is the timedependent flux of atoms emerging from the grain. Finally, the flux of released atoms is the arrow leaving the box after passing the vertical line on the right. In either box the outgoing flux $\phi_{\text {out }}$ is expressed as a function of the incoming flux $\phi_{\text {in }}$, the relevant release probability function $R$ and of the decay constant of the nucleus $i$ as follows:

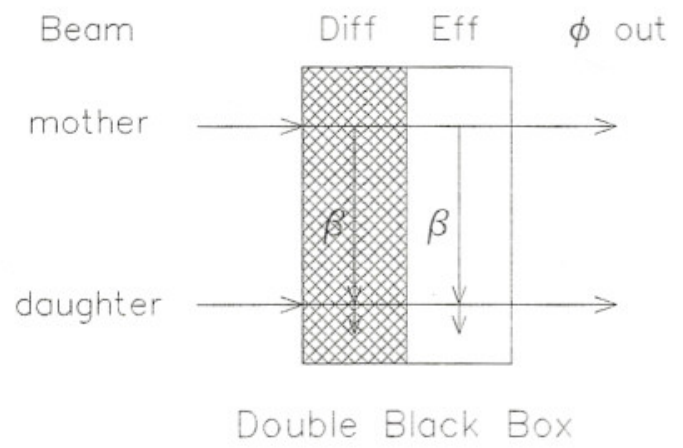

Fig. 1. Schematic model of sequential diffusion and effusion (left to right arrows) including $\beta$ decay in the target (vertical arrows). Creation rate by fission, crossing the surface of grains and leaving the target are represented by vertical lines, from left to right. $\phi_{\text {out }}\left(t^{\prime}\right)=\int \phi_{\text {in }}\left(t^{\prime \prime}\right) R\left(t^{\prime}-t^{\prime \prime}\right) \mathrm{e}^{-i\left(t^{\prime}-t^{\prime \prime}\right)} \mathrm{d} t^{\prime \prime}$.

The exponential accounts for the chance the nucleus of interest has to survive decay at $t^{\prime}$ after being created at $t^{\prime \prime}$. Whereas the nucleus can be lost by radioactivity, decay of the mother nucleus adds another contribution to be included in $\phi_{\text {in }}\left(t^{\prime \prime}\right)$,

$\phi_{\beta}\left(t^{\prime \prime}\right)=\lambda_{\mathrm{m}} n_{\mathrm{m}}\left(t^{\prime \prime}\right)$,

with $n_{\mathrm{m}}\left(t^{\prime \prime}\right)$ the number of mother nuclei with decay constant $\lambda_{\mathrm{m}}$ present in the box, see [6] for details.

Finally, one obtains three different functions contributing to the flux out of the target, one proportional to the in-target independent production rate of the nucleus of interest, two proportional to the cumulative production rate of the mother.

The calculations in [6] have been carried out with the general form for diffusion given as

$D(t)=\sum a_{k} \mu_{k} \mathrm{e}^{-\mu_{k} t}$,

which is normalised by $\sum a_{k}=1$. This form contains the particular expressions derived by Fujioka [7] for simple geometries, e.g. for spherical grains the correspondence is $\mu_{k}=k^{2} \mu_{0}$ and $a_{k}=6 /(k \pi)^{2}$. Effusion is simply described by a single exponential function with constant $v$

$E(t)=v \mathrm{e}^{-r t}$.

\subsection{Release efficiency}

It is of interest to explicit the formulae for release efficiency. One takes the flux at the end of a long irradiation, letting the irradiation time $t_{\mathrm{ir}}$ to go to infinity. The limits of the three flux functions, for fission only $\left(\phi_{\mathrm{F}}\right)$, decay during diffusion $\left(\phi_{\mathrm{D} \beta}\right)$ and decay during effusion $\left(\phi_{\mathrm{E} \beta}\right)$, become

$\phi_{\mathrm{F}}\left(t_{\mathrm{ir}} \rightarrow \infty\right)=p \varepsilon_{\mathrm{D}} \varepsilon_{\mathrm{E}}$,

$\phi_{\mathrm{D} \beta}\left(t_{\mathrm{ir}} \rightarrow \infty\right)=p_{\mathrm{m}} S_{\mathrm{Dm}} \varepsilon_{\mathrm{D}} \varepsilon_{\mathrm{E}}$,

$\phi_{\mathrm{E} \beta}\left(t_{\mathrm{ir}} \rightarrow \infty\right)=p_{\mathrm{m}} \varepsilon_{\mathrm{Dm}} S_{\mathrm{Em}} \varepsilon_{\mathrm{E}}$,

These formulae are very simple. Diffusion and effusion involve a factor $\varepsilon$, while decay involves its complement $S=1-\varepsilon$. This relationship is logical since the flow must be conserved in the saturation limit. Thus we only need to give the expressions

$\varepsilon_{\mathrm{D}}=\sum_{k} a_{k} \frac{\mu_{k}}{\mu_{k}+\lambda}, \quad \varepsilon_{\mathrm{E}}=\frac{v}{v+\lambda}$.

which, in fact are the well-known efficiencies for diffusion and effusion.

The decay functions $\phi_{\mathrm{D} \beta}$ and $\phi_{\mathrm{E} \beta}$ differ from the fission function $\phi_{\mathrm{F}}$ only from the in-target production rate, $p$ or $p_{\mathrm{m}}$, and through insertion of a $S_{\mathrm{m}}$ term depending on the release of the mother nucleus. The behaviour at the limits can be understood intuitively. If the mother diffusion or effusion time is much shorter than the radioactive half-life, the mother nucleus has a large chance to be released before 
decay ( $S$ is small). Consequently, the corresponding function vanishes and there is nothing to be added to $\phi_{\mathrm{F}}$. In the other limit, most of the mother nucleus shall decay ( $S$ is close to 1). If this happens during diffusion $\phi_{\beta}$ merges with $\phi_{\mathrm{F}}$, but now with the scaling factor $p+p_{\mathrm{m}}$.

The interest of this formalism is that it allows to incorporate a realistic fraction of the mother production, being controlled by the interplay of its release parameters and lifetime. The term mother can also apply to an isomer in the nucleus of interest, in which case the release parameters for mother and nucleus of interest are the same.

In order to compute the contribution of the mother nucleus one needs its release parameters. It is, however, little realistic to hope to extract all the parameters from a fit of release efficiency and one must rather guess the release parameters of the mother element from other experiments. A further simplification is almost unavoidable. One considers the extreme cases only in which one of the processes is fast enough so that $\beta$ decay cannot occur during it. This corresponds to smashing the box of the fast process. An approximate way to keep both processes within one-box is to use a global release function in which $D$ and $E$ have been convoluted from the beginning.

$R(t)=\sum a_{k} \mu_{k} v \frac{\mathrm{e}^{-\mu_{k} t}-\mathrm{e}^{-v t}}{v-\mu_{k}}$.

This keeps the general form given for $D(t)$ with an extra coefficient of opposite sign representing effusion.

We explicit as an example the quite common case of a nucleus having an isomer, while the parent has none. This situation can occur for instance in the study of even-mass alkalis, whose parents have $0^{+}$ground states. Both the ground-state ( $\mathrm{g}$ ) and the isomer (i) are fed by decay of the mother $(\mathrm{m})$. The observed yields y are

$$
\begin{aligned}
& y_{\mathrm{i}}=n I_{\mathrm{p}} \varepsilon_{Z}\left(\sigma_{\mathrm{i}}+\sigma_{\mathrm{m}} S_{\mathrm{m}} b_{\mathrm{m} \rightarrow \mathrm{i}}\right) \varepsilon_{\mathrm{i}}, \\
& y_{\mathrm{g}}=n I_{\mathrm{p}} \varepsilon_{Z}\left(\sigma_{\mathrm{g}}+\sigma_{\mathrm{m}} S_{\mathrm{m}} b_{\mathrm{m}-\mathrm{g}}+\bar{\sigma}_{\mathrm{i}} S_{\mathrm{i}} b_{I T, \mathrm{i} \rightarrow \mathrm{g}}\right) \varepsilon_{\mathrm{g}} .
\end{aligned}
$$

Here $n$ is the number of target nuclei per surface unit, $I_{\mathrm{p}}$ the particle current, $\varepsilon_{Z}$ the efficiency of separation except for the dependence on isotope lifetime, $\sigma_{\mathrm{m}}, \sigma_{\mathrm{i}}$ and $\sigma_{\mathrm{g}}$ are the cross-sections to populate the corresponding states in fission, factors $S=1-\varepsilon$ are defined above and $b$ 's are decay branchings. The last factor $\varepsilon$ is the release efficiency for the nucleus of interest in its particular state. The quantities within brackets can be regarded as effective cross-sections. In the second equation $\overline{\sigma_{i}}$ is such a term, namely the one in the first equation. The fitting method is iterative, in the sense that the 'experimental' release efficiency $\varepsilon$ is not fixed forever but varies according to the current values of the trial release parameters, e.g. here both $S_{\mathrm{i}}, \varepsilon_{\mathrm{i}}$ and $\varepsilon_{\mathrm{g}}$ are functions of the unknown release parameters of the element of interest. We note that cross-sections relevant to our work at IRIS, Gatchina [2] have been measured for $1 \mathrm{GeV}$ protons on uranium owing to comprehensive work at GSI [8]. However, that experiment cannot distinguish whether the recoiling nucleus is in its ground state or not. The dis-

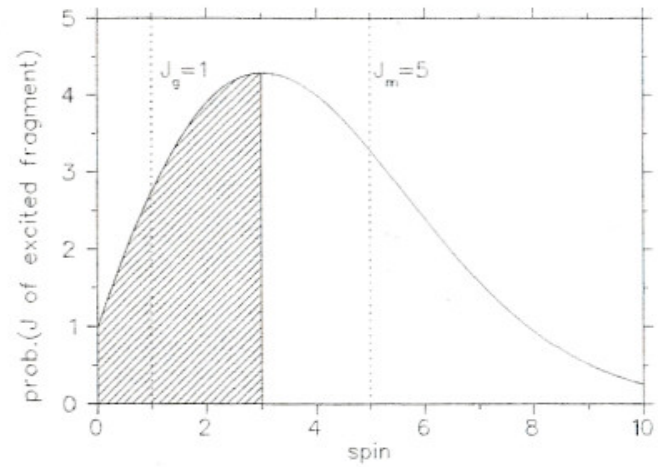

Fig. 2. An estimate of the relative feedings into 2 long-lived states, here one of spin 1 and the other of spin 5, is obtained by assuming a distribution of population of highly-excited fragments versus spin (solid line) and considering that the hatched area gives the probability of feeding the state of lower spin (from [9]).

tribution of population among the isomer and ground states is not known. In the absence of any other data we have relied on estimates using a simple model developed for thermal fission [9]. The principle of calculation is shown in Fig. 2. It is obvious that the validity of this approach in high-energy fission needs to be proven.

\section{Comparison with experiments}

In most cases, decay in the target results in an apparently slower release. These cases can be treated by using a superposition of exponentials, which corresponds formally to the model with the sole diffusion box. However, a fast component appeared in a release curve of ${ }^{91} \mathrm{Rb}$ whereas it was absent in the release curve of ${ }^{86} \mathrm{Rb}^{\mathrm{m}}$ (only fed via fission) recorded under the same target conditions. This could be explained only by explicitly separating the intarget decay probabilities during diffusion and effusion. The fast component is due to $\beta$ decay of ${ }^{91} \mathrm{Kr}$ during effusion, which by-passes the slow diffusion of rubidium [6].

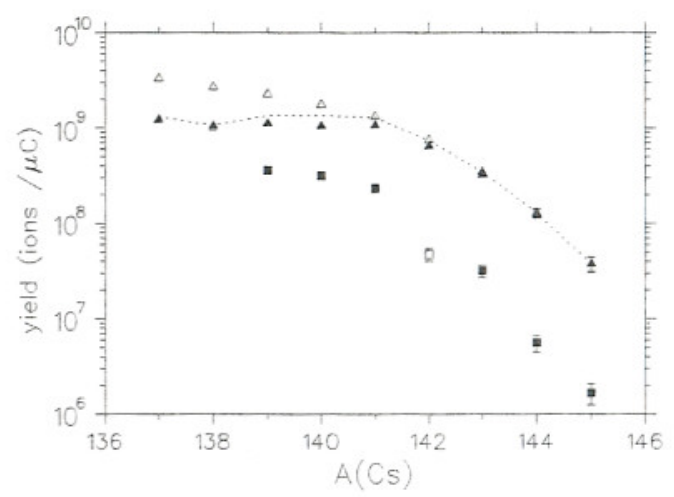

Fig. 3. Exampie of in-target and experimental production rates of Cs isotopes. The former are calculated considering population by fission only, i.e. assuming $\mathrm{Xe}$ is very quickly released (full triangles), adding decay of Xe fully (empty triangles) and partially under assumption of an empirical release function for $\mathrm{Xe}$ (dotted line). Squares show the experimental yields. 

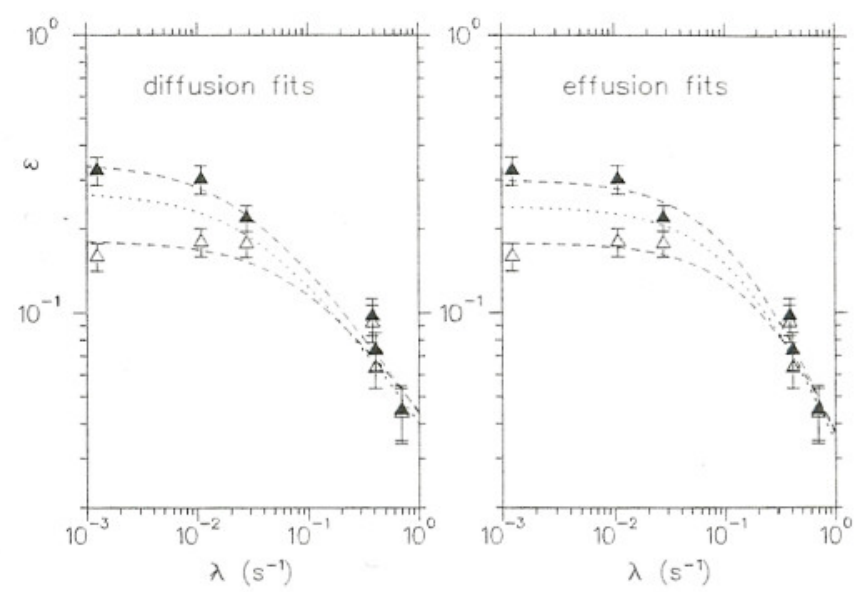

Fig. 4. Fits of efficiency, i.e. the ratio of measured to calculated in-target yields, versus decay constant for $\mathrm{Cs}$ nuclei from the above example, where from ${ }^{142} \mathrm{Cs}$ has been removed. Symbols corresponds to those in Fig. 3. For the presumably 'more realistic' Xe release time only the fit is shown for clarity of reading.

An example of release parameters extracted from an efficiency curve shown in Fig. 3 demonstrates the difficulties inherent to such an analysis. First, there is a problem with the yield of ${ }^{142} \mathrm{Cs}$ which is always calculated to be too low [2] when using $\gamma$ spectroscopy and must be removed from the data set to be fitted. Moreover, our release curves do not have the standard shape for either diffusion, effusion or their convolution. The use of these formulae is only justified by the simplicity and the purpose of quick comparison of different experimental conditions.

The difference of calculated in-target production rates causes noticeable changes of the overall efficiency curve versus nuclear lifetime. As shown in Fig. 4, a longer release time of the Xe mother (empty symbols) decreases the calculated overall efficiency for long-lived Cs nuclei and results in an apparent faster release of caesium.

The $T_{\mu_{0}}=\ln (2) / \mu_{0}$ values for pure diffusion obtained without (resp. with full) parent feeding are $22.5 \mathrm{~s}$ (resp. $6.8 \mathrm{~s}$ ), while the corresponding $T_{v}$ values for effusion are $5.0 \mathrm{~s}$ (resp. $2.6 \mathrm{~s}$ ). 'More realistic' values of $17.0 \mathrm{~s}$ (resp. $4.2 \mathrm{~s}$ ) are obtained with an arbitrary exponential release function ( $10 \mathrm{~s})$ of Xe. In all cases, the relative errors derived from the fitting method are typically $30 \%$. On the basis of the $\gamma^{2}$ minimum values there is no possibility to favour a release parameter for Xe. Finally, $\chi^{2}$ values neither definitely establish which of diffusion or effusion is the slowest process. This, however, becomes possible when measuring nuclei with even shorter lifetimes than shown here [10].

\section{Conclusion}

We have investigated the influence of decay in the target when extracting information on the release of fission products from ISOL targets. The limiting case of a steady beam situation provides a simple correction to the cross-sections to be used in order to calculate the release efficiency. Determination of release parameters from the variation of efficiency versus nuclear lifetime is simple but, unfortunately, is poorly suitable for fission products since the parameters needed for the parent nucleus are generally not available under the same target conditions. So far, its seems appropriate to use it to compare different target conditions, but hardly to conclude about the dominant mechanism of release. All in all, a consistent approach of analysis of release curves and efficiencies is necessary to make sure that the deduced parameters have a physical meaning.

\section{Acknowledgements}

We acknowledge the financial support of the European Community under the FP6 "Research Infrastructure Action-Structuring the European Research Area" EURISOL DS Project Contract No. 515768 RIDS. The EC is not liable for the use that can be made of the information contained herein. For PNPI this work has been supported by ISTC Project No. 6529.

\section{References}

[1] R. Kirchner, Nucl. Instr. and Meth. B 204 (2003) 179, and references therein.

[2] A.E. Barzakh et al., Activity report of the PLOG-collaboration, available on EURISOL web site: <http://www.eurisol.org/siteol/taskdetails.php>

[3] A. Bracco, A. Pisent, LNL-INFN (REP) 181/02, 2002.

[4] http://www.ganil.fr/research/developments/spiral2/index.html.

[5] See http://www.eurisol.org/site01/index.php.

[6] G. Lhersonneau et al., Nucl. Instr. and Meth. A 566 (2006) 465.

[7] M. Fujioka. Y. Arai, Nucl. Instr. and Meth. 186 (1981) 409.

[8] M. Bernas et al.. Nucl. Phys. A 725 (2003) 213.

[9] D.G. Madland, T.R. England. Nucl. Sci. Eng. 64 (1977) 859.

[10] B. Roussière et al., Nucl. Instr. and Meth. B 194 (2002) 151. 